\title{
DENOMINAZIONi Di LUOGO INTITOLATE A EDIFICI DI CULTO, MONASTERI, ORATORI, ALTARI E ALTRI MANUFATTI LEGATI ALLA PRESENZA DEL SACRO
}

\author{
Alfonso Germani \\ Centro Inchieste Dialettali “La Ciocia”, Arce, Italia
}

\begin{abstract}
Place names referring to places of worship, monasteries, oratories, altars and other objects related to the presence of the sacred
\end{abstract}

\begin{abstract}
Place names identifying temples, churches, cults, beliefs, superstitions, rites and symbols are found in European toponymy, above all in Italy, France, Spain, Portugal and Austria. This contribution contains a selection of Italian and French geographical names of Christian origin that refer to spaces (churches, chapels, cells, cloisters, monasteries, oratories, altars and other objects), symbols and events and that traditionally have the function of marking sacred places.
\end{abstract}

Keywords: sacred places, religious buildings, sacred toponymy.

\section{Introduzione}

I nomi di luogo intitolati a edifici di culto, basiliche, chiese, monasteri, cappelle, celle e altri manufatti legati alla presenza del sacro, hanno segnato profondamente la toponomastica del continente europeo e costituiscono un dato ineliminabile del suo patrimonio culturale e storico.

Le forme arcaiche cristallizzatesi nel tempo della toponomastica religiosa, come scrive la Prof. Alda Rossebastiano (2007), "possono fornire dati rilevanti per la ricerca dialettologica", poiché attestano fasi superate nei dialetti attuali; ciò vale in particolare per il territorio della Francia, dove moltissimi toponimi hanno conservato più o meno intatte le originarie forme dialettali che caratterizzavano le regioni di appartenenza.

Nel corso del Medio Evo, mentre la cristianizzazione lentamente si estendeva, si delinearono a poco a poco i luoghi in cui il culto si fissava e la scelta dei nomi comuni usati per designare e qualificare gli edifici religiosi, variamente caratterizzati.

Tra le denominazioni più antiche vanno ricordate: BASILICA, DOMUS, DOMUS DEI, DOMUS ORATIONIS, DOMUS COLUMBAE, ECCLESIA, ALTARE, ORATORIUM, CAP(P) ELLA, MONASTERIUM, CELla, ABBATIA, CANONICA, PLEBS, PAROCHIA, DOMINICUM, APOSTOLIUM, MARTYRIUM, DELUBRUM, FANUM, TEMPLUM.

Sono questi i nomi, i loro derivati e i loro composti che, insieme ai molti nomi di santi titolari delle chiese, costituiscono quasi tutta la toponomastica sacra di paesi come la Francia, la Spagna, l'Italia, il Portogallo, l'Austria, ecc.

In un contributo congressuale necessariamente breve non è possibile affrontare 
un tema così vasto e importante come quello dei toponimi di origine cristiana, non dico per l'intero territorio europeo ma neppure per un gruppo ristretto di nazioni, per cui mi limito a presentare una selezione di forme usate per designare i luoghi di culto in Italia e in Francia.

\section{Ecclesia-Basilica}

In Italia gli oggetti toponomastici appartenenti alla categoria ${ }^{1}$ 'appropriazione dello spazio di tipo religioso o funerario' (abbazia, basilica, campanile, cappella, cattedrale, cella, chiesa, convento, cripta, croce, ecc.), sulla base della cartografia IGMI, fogli 1: 100000 sono $23514^{2}$.

Sulla base dei documenti più antichi e le conferme di successive attestazioni, gli studiosi hanno parlato di competizione lessicale panromanza ${ }^{3}$, di uso plurisemantico dei termini (De Santis 2009: 117) o di termini sinonimi (Aebischer 1965). Proprio quest'ultimo linguista, in un suo fondamentale contributo, sull' antécedent d'ecclesia sur basilica, pubblicato nella miscellanea in onore di A. Schiaffini, scriveva:

Durante centinaia di anni, i territori che conoscono ancora o hanno conosciuto BASILICA con il significato di chiesa, siano essi l'Africa del nord, l'Italia settentrionale, la Dacia, la Gallia e la valle del Reno, la penisola iberica e la Sardegna, hanno conosciuto ugualmente ECCLESIA con lo stesso significato. Sulle iscrizioni in Africa, per non citare che un esempio, i due termini si equivalgono. [...] Il più grave errore di metodo che si può commettere, studiando il binomio ecclesia-basilica, è ritenere che da sempre, per così dire, dall'inizio del cristianesimo, ciascuno dei due termini abbia avuto la sua area di utilizzazione [...]. Al contrario, tanto nel greco che nel latino ecclesiastico, essi all'inizio hanno dovuto lottare contro dieci o venti altri concorrenti per riuscire a imporsi (Aebischer 1965: 12).

A confermare che i termini basilica-ecclesia potevano essere sinonimi ci sono dei documenti relativi alla chiesa regia di S. Giovanni in Monza, designata nell'anno 768 come ecclesia e l'anno seguente come basilica. "Che BASILICA fosse in altri tempi più diffuso in territori che ora conoscono come nome comune solo derivati di EC(C)CLESIA è provato dalla toponomastica dell'Italia e della Francia” (Tagliavini 1982: 274).

\section{Basilica}

Ling.- BASILICA, gr. (stoà) basiliké, con il sign. di '(portico) 'regio'; preso sostantivamente, designò, nella Roma pagana, un edificio sontuoso dove i magistrati amministravano

\footnotetext{
1 In base al glossario degli indicatori geografici redatto da Laura Cassi (vedi Cassi e Marcaccini 1998).

2 La maggiore numerosità degli oggetti/segni religiosi-toponimo si riscontra al nord (Fuschi 2008: 299).

3 La competizione lessicale panromanza, con significative appendici celtiche e albanesi, tra BASILICA /ECCLESIA/ CAPPELLA/ ORATORIUM è testimoniata da numerosi toponimi soprattutto in Francia, Svizzera romanda, Grigioni, Italia padana, Friuli.
} 
la giustizia. Per un'evoluzione del significato, il termine è passato dal V sec. a designare un edificio cristiano consacrato al culto, una chiesa, talvolta una semplice cappella ${ }^{4}$.

«Avec cette signification chrétienne, le mot BASILICA se conserve dans les régions de Rhétie et de Véglie et dans la langue roumaine à la différence des autres langues romanes, qui emploient ECCLESIA. Encore un cas où la langue roumaine se montre même plus conservatrice que la langue italienne» (Pop 1966: 628).

In Italia incontriamo queste forme:

Basilica Goiano, Montechiarugolo (PR), Basilicanova (PR), Basaglia (varie), Basalghelle (Mansuè-TV), Basaluzzo (AL), Baseleghe, S. Michele al Tagliamento (VE), Trebaseleghe (PD), Baselga di Bondone (TN) e altre, Baselica di Valtaro (PR) e altre, Basereca (PR), Baserica (PR), Basirica (PR), Baslenga (PR), Basèlice (BN).

Secondo Jacques Moreau, il termine BASILICA in Francia venne dato, soprattutto ad Ovest, al centro e a Nord, alla fine dell'epoca romana e nell'Alto Medio Evo alle prime chiese parrocchiali e alle chiese dei primi monasteri (Basialgues, Bazalgue, Bazauges, Bazouges etc.) (Moreau 1972: XVII).

Tra le più antiche forme di BASILICA vanno segnalate:

Bazoges en Paillers (Vendée, Baselgius 1056), La Bazoge (Mayenne, XI sec.), La Bazoque (Orne, Basogia 1229), La Bazeuge (HV, < baseuga XII sec.), Bazaiges (Indre, Baseajes 1200, Besselgia 1212), Bazèque (PdC, Basilica 1098), (La) Bazouge (Mayenne, Ille et V.), La Baroche sous Lucé (Orne, Bazocha 1150, oil basoche 'église', croisé avec paroche 'paroisse') (Nègre 1991: 1504-5 e Gendrom 2003).

\section{Ecclesia}

L'it. chiesa e il fr. église continuano il greco ekklesia 'assemblea'; i cristiani l'applicarono propriamente alle loro assemblee (comunità dei cristiani), in seguito designò ogni locale dove le comunità si riunivano per la preghiera e per assistere alle sacre funzioni. Questo secondo significato di église è attestato, verso la fine del VI secolo, dagli scritti di Grégoire de Tours e di Fortunat.

Église è rappresentata oggi dal nome di almeno una trentina di frazioni (écarts) in tutta la Francia. Rispetto ai toponimi italiani dove le forme e varianti di chiesa non hanno una rilevante discendenza (Chiesa, Chiesina, Chiesino, Chiesuol, Chiesuola e poche altre), in Francia è più marcata e di gran lunga più ampia la serie di forme toponomastiche corrotte: egleise, iliz e lan, domerie, illièse, élize e élise, kerke e kerque, chilche, lheïza, eliça, eliza, elicha, elissa e via elencando.

Tra le prime rare menzioni anteriori all'anno mille, si segnalano: Égriselles-leBocage (Yonne, Aecclesiolae, IX s.) et Neuvéglise (CA, Nova Ecclesia, 929).

4 BASILICA «désignait en latin médiéval un monument érigé en mémoire d'un martyr et généralement dépositaire de reliques» (Lejeune 2002: 83). 
Sono dei secoli successivi:

Neuve-Église (BR), Neuglise à Bessay-sur-Allier (AL, Nova Ecclesia, 1373), Neuf-Église (PdD, Nove Yglise, 1401), Égliseneuve sur Billom (PdD, Gleyzanova, 1401). D’autres adjectifs sont visibles dans: Blanche-Église (Mos., Alba Ecclesia, XII s.), Manéglise (SM, Magna Ecclesia, 1155), 'grande église', Vieille-Église (YV, Terris Veteris Ecclesiae, 1248) (Gendrom 2003: 144-145 et al.).

Nel Nord della Francia incontriamo forme fiamminghe:

Dunkerque (Nord, Dunkerka, 1067, TWB) fiamm. duin 'duna, collina' + kerk 'chiesa'; Nortkerque (PdC, Northkerke v. 1119, VTF 342), fiamm. noord + kerk $=$ chiesa del nord; Zutkerque (PdC, Zuitkerka, 1088-89, DR, Sutkerka, 1123, TWB), fiamm. zuid 'sud' + kerk.

In Alsazia è presente il germ. kirche, preceduto da un determinante come p.e. in: Altkirch, dipartimento Alto Reno; il top. deve la sua origine e il nome a una église-mère (avente degli annessi) che la tradizione fa risalire ai primi tempi del cristianesimo (Aldechiarcum, XI s., Altikirch = germ. agg. alt (= vieille) + kirche, cfr. con Vieille-Église, $\mathrm{SO}$ e H. Savoie). Suoi simmetrici sono: Neukirch, l'attuale Neuve-Église (BR), apparentato a Neuenkirch annesso a Friesenheim (BR), santuario mariano e luogo di pellegrinaggio dell'Alsazia centrale; Neukirch (TG), 'nuova chiesa', centro della Svizzera settentrionale; Neuenkirch (Lucerna), 'la nuova chiesa,' 'ancien couvent de nonnes'; Neukirch, in Germania, denominazione di quattro comuni in circondari del Baden-Württemberg, Sassonia e Renania-Palatinato (Schweitzer 2001: 39 et al.).

\section{Monasterium}

In it. var. ant. Monesteri $[<$ lt. tardo eccl. Monasteriu(M) dal gr. monastếrion, 'dimora solitaria', derivato di monastếs 'monaco'; prima metà sec. XIII, "cella dell'eremita"].

Il Pellegrini, per l'Italia, riporta queste antiche forme di monastero: Mosterio, Mustiolo/Mustollio,-olio, Monistero, Monisterino, Monasterolo, Monastirolo, Monastier (Pellegrini 1990: 224).

In Francia, nei secoli XI-XII, i principali centri religiosi erano monasteri o abbazie. Gli a. fr. moustier e moutier e il prov. monestier sono i termini più usati nel Medio Evo per designare l'église del villaggio, essi derivano da una forma lt. alterata ${ }^{\circ}$ MONISTERIUM. Il fr. attuale monastère (1279) è una formazione dotta, come anche l'it. monastero (sec.XIV), erroneo sing. rifatto sul pl. monasteri (DISC: s.v.).

A proposito di église e moutier, termini tutti e due vitali in Francia nel XII secolo, che spesso compaiono affiancati nello stesso testo come sinonimi, sempre l'Aebischer nel citato contributo osserva che "la scelta di moutier per église si è prodotta in un'epoca relativamente recente" (Aebischer 1965: 11).

Alla fine del Medio Evo, la parola mou(s)tier aveva il significato quasi esclusivo di 
'église' e conserva oggi questo significato nelle parlate della Vallonia, della Lorena, della Franca-Contea e della Svizzera romanda.

Ecco alcune forme regionali per monastère: Lan-, Lean- ${ }^{5}$; Moustier(s), Mostier, Mouterre (Vn, 1383, Monter, 1449 Moustier), Moûtier, Montier, Monastier, Monestier, Monestiés, Monétier, Motey, Monetoy ${ }^{6}$.

\section{MONASTERIUM + agg.:}

v. fr. major, majour, "plus grand": Marmoutiers (BR, IL); VI s., "in monasterio vero, qui nunc Major dicitur, basilicam in honore sanctorum apostolorum Petri et Pauli aedificavit"; 881-882, "abbatiam Majoris Monasterii” (Vincent 1937, par. 872); fr. neuf, "nouveau": Neufmontiers (S-et-M.); 1210, "Novum Monasterium"; 1222-43, "apud Novum Monasterium", neuf + moutier, neuf, compris comme le numéral, a entraîné -s du pluriel (Nègre 1991, s.v.).

Nomi germanici + moutier:

Bon Moutier (M.e.M., cella quae nuncupatur Bodonis Monasterium, 816= NP germ. BODONUS+ MONASTERIUM).

Suffissi diminutivi:

-EL: ${ }^{*}$ MONASTERELLUM > Ménestreau (NV, 1174 Monesterellum), Menetreau (BC, 1178 - Monestellum, 1237 Monesterellum), Ménétreux (CO, 1193 - Monesterel, 1281 Monestereul), Montrieux (L, Ch, 1369-70 - de Monasterello in Gaudo); -ELET: Montrelet (Som, 1118 - Monstrelet, 1171 Mostrelet); -ET: Le Moutaret (Is, XIII s. Mostaret), Montret (appartiene senza dubbio alla stessa serie, anche se una falsa etimologia lo apparenta a mont); -OLU > ÒL (occitano), MONASTERIOLUM > Ménestérol (Dord.), Ménestrol (Dord.), Ménétréol (PdD), Ménestreau (L, Nièvre), Ménétreuil (SL), Ménétru (Jura), Monistrol (HL), Montrol (HV), Montrollet (CH).

5 Leandi, Leanti; Langoat (CdN, bret. lann 'monastère, sanctuaire, église') + coat, coet 'bois' - Nègre 1991 s.v.), Minihi (CdN, bret. minic'hi 'monastère' - Nègre 1991, s.v.); Monac'hti, Mouster (Bretagne), Monêtier, Monestier (Ubaye), Munastèri, Mounesté (Provence), Mouterot (petit monastère) (Franche-Comté), Münster (Alsace) (Nègre 1991).

6 Moutier, forma prevalente, era in lingua d'oil, il nome comune per monastero: Les Trois Moutiers, Les Moutiers-Hubert (Calv. 1155), var. Montier, come in Montier-en-Der (HM, Monasterium Putiolos in vasta Dervi, 692), Montiermoyen (Bourges, Cher, 1152 Mediano Monasterio); Monastier: Le Monastier (HL, Monasterium Sancti Petri nell' 840, Villa Monasterii nel 987), var. Le Monestier-de-Clermont (IS, XIII s.), Monestiés (Tarn, 961, Illo castello de Monasterio); Le Monétier-Les-Bains (HA, XI s., Monasterium), Monstiers (BA, 1125, ecclesiam castri quod dicitur Monasterium); Montiers (Oise, 1164, villae quae dicitur Monasteria), Monthières (Bouttencourt SOM, 1185, Monsterri); Motey (HS, 1133, de mosterio; Monetoy (Écuisses SL, 1320, ecclesia de Monetay) (Fénié e Fénié 2003: 95 et al.). Moutier, Mostier, Motier, Mothy sono presenti anche nella Svizzera romanda (Jaccard 1978: 298). 


\section{Altaria}

Nell'antichità, "edicola per il culto degli dei" (dal pl. ALTARIA, che gli antichi intesero comp. dall'agg. f. ALTA + ARA: "altare autem ab altitudine consta esse nominatum quasi alta ara" - Isidoro 15-4-14, Battaglia 1961 s.v.); nelle chiese, "tavola sulla quale il sacerdote celebra la messa".

It. altare, s.m. (ant. anche f.); it. a.: altari (ante 1333, Deon.), altare (n.f.), autare (ante 1406); gen. otar (sec. XIV), piem. alter (1200 ca); con cambio di suff.: pis. artale, laz. sett. lartale. Altre lingue e dialetti: romeno altar, vegl. altúr, friul. altâr, eng. utel, fr. a. altier, fr. autel (sec. XII), occit. a. autar (1150), sp. otero ${ }^{7}$, sardo altare, merid. autaru. È penetrato diffusamente nelle lingue contigue: alb. eltér, ilter, letér; slavo a. oltara, bulg. oltar, a. ted. a. altari.

È presente nella toponomastica italiana con queste forme:

Altare (SV, forma dial. Laté con l'art. concresciuto), L'Altare (FI), Piana Altare (Cercemaggiore - CB), Monte Altare (Vittorio Veneto - TV), Colle Altare (Usseglio - TO), C. Altare (S. Margherita di Belce - AG), Passo Altar (Spiazzo - TN), Pietra dell'Altare (Sgurgola - FR, Pedace - CS), Pian(o) dell'Altare (Ruota Capannori - LU, Pontremoli - MC, Ripacandida - PZ), Sa Pedra de S'Altare (Birori - NU), Artale (Cune Borgo a Mozzano e Pescia - PT, Furci Siculo - ME), Tre Artale (Tralia - PA), P. te dell'Artale (S. Margherita Ninfa - TP), Altariól/Altaról, Altariolo (< ALtARIolo 'tabernacolo'), presenti nel Friuli, Triól de la Cros (Cencenighe - BL), lad. ven. Horto all'Altriol, lad. at. L’Ateriol, Còl de Auteriól (Colle S. Lucia), lad. cad. Altariol (ampezz.) (DT e altre fonti).

Francia: «Fr. autel (1080), est une altération de l'anc. fr. alter ${ }^{8}$ (1050) et var. altier, emprunté au lt. religieux ALTARE, n. m. du pl. cl. ALTARIA, désignant un support placé sur la table des sacrifices, sur l'autel au sens antique (ARA)» (DHLF s.v.), dove si depositavano le offerte e si offrivano i sacrifici agli dei. Ha assunto ben presto il significato di église d'ordre secondaire, come attesta un passo del capitolare di Carlo il Calvo9.

Questi toponimi ricordano un altare precristiano:

Les Autels (Aisne, 1190 Altaria; Calvados, 1024), Lautier (Vn 1093 Ecclesia sancti Leodegarii de altario, 1184 Ecclesia de altari), Col de l'Autaret (1042 Altaretum, Monte Delfinato, sulle Alpi Graie, al confine italo-francese).

7 Top. leon. a. Autairo, spagn. a. Auteiro, Autero, Otero.

8 Anc.fr. alter, -el (1150, Péler. Charl; lat. ALTAREM), 'table où l'on célèbre la messe', dim. autelet (1306, Guiart) (Greimas s.v.). «Le changement de la finale -ER (Alexis, strophe 34, éd. Paris et Pannier, Paris, 1872:147 «Deus fist l'imagene por soe amor parler. Al servitor qui serveit al alter» en -EL est dû prob.à une substitution du suff. -EL à la finale -ER rare dans des termes désignant des objets concrets (cette substitution s'explique peut-être par une attraction paronymique avec OSTEL 'hôpital, hôtel' à une époque où les Hôtels-Dieu étaient des maisons religieuses)». (cnrt.fr./definition/Autel?).

9 Capitolare di Carlo il Calvo dato a Tolosa nel giugno 844: "Si necessitas populi exegerit, ut plures fiant ecclesiae, aut statuantur altaria, cum ratione et auctoritate hoc faciant” (Longnon 1920-1929, par.1408, p.346). 
Questi altri indicano la presenza di una chiesetta o di un oratorio:

(Les) Autet(s) (Aisne, Calvados, EL, HS), Les Autels, Cinq Autels (Calvados, Ecclesia de Quinque Altaribus, v.1350), Autheux (Somme, 1150 o 1190), Les Authieux ${ }^{10}$ (Eure, Orne, Calvados, SM), Lauthiers (V) (Vial 1983: 224-225 et al.), Belhôtel (Orne, de Bello Altare $=$ bel + autel $)$.

Zoteux (Somme) et Zoteux (PdC, Altaria 1142), répresentent également, les pluriels 'les auteux' ayant été compris 'zauteux' et article le supprimé (Gendrom 2003: 145).

\section{$\operatorname{Cap}(\mathbf{p})$ ella}

It. Cappèlla, s.f., edificio di culto di piccole dimensioni e isolato; ambiente inserito in un castello, annesso a una chiesa, a un cimitero; tabernacolo, edicola religiosa, nicchia votiva o commemorativa con immagine sacra spec. posta sulle vie. [lat. mediev. CAPPELLA, dim. di CAPPA, propr.luogo (oratorio dei re merovingi), in cui si conservava una reliquia della cappa di s. Martino di Tours] (Treccani online). Ecco alcuni toponimi italiani:

Cappella (NA), Cappella Cantone (CR), Cappella de' Picenardi (CR), Cappella Maggiore (TV), Cappelle (comuni e frazioni, varie), Cappelle sul Tavo (PE).

In Francia, «bientôt CAP(P)ELLA désigna le lieu même où, dans le palais, était conservée la chape de Saint Martin, et c'est dans ce sens qu'il faut entendre le surnom de la ville d'Aix-la-Chapelle (appelée Aix avant sa construction), où l'on sait que les rois avaient un palais: Carles sera ad Ais a sa capele lit-on dans la Chanson de Roland» (Longnon 1920-1929: 349).

È presente nel nome di più di cento comuni (Vial 1983: 225) sotto diverse forme:

La Chapelle (AL, Ard., Char, Morb., Som., SM, Jura, Marne, V); La Chapelle, Savoie (franc. del frp tsapela 'chapelle'); La Capelle (PdC, Aisne, Lozère, Av., CA, TG, Gard, Aude, Eure); Lacapelle (CA, Lot, TG, LG); Les Chapells (HL, Capellae, v. 1198, pl.) (Nègre 1991: 1506 et al.);

nei dialetti normanno, piccardo e vallone:

Capelle (Nord); Capelle-les-Grands (Eure, Capellas, 1025, Capelles les Grans, 1414; pl. di oil capelle + agg. granz, f. pl.: 'les grandes chapelles'; il s'agit prob. d'une seule église qui avait plusieurs chapelles latérales et c'est pour cela qu'on a supprimé -s final de Capelle(s) (Nègre 1991: 1505); Cap(p)elle (Nord, PdC, Aude) (1122, apud Capellam) o a Wemaers-Cappel (Nord) con il nome del fondatore Winimar (Nègre 1991 et al.),

10 Les Authieux est à rapprocher de tieux, qui fut une des formes du pluriel de l'adj. indéfini tel. 
con l'articolo fuso alla parola:

Lachapelle (LG, HM);

con gli aggettivi:

La Haute Chapelle (Orne, Alta Capella, v. 1050); Neuve Chapelle (PdC), La Chapelle Neuve (CdN); Vieille Chapelle (PdC); La Chapelle Blanche (CdN, IL, Capella Alba); La Chapelle Blanche (Savoie, Blancha Capella, v. 1100), Grandes Chapelles (Aube, oil grandes + chapelles); La Chapelle Graillouse (Ardèche, Capella grailosa, 937, nord-occ. chapèla + gralha 'corneille' + suff. adj. -osa 'chapelle pourvue de corneilles'), La Chapelle Largeau (Deux Sèvres, Capella Largea, 1269, oil chapelle + peut-être la rejau, variante poitevine de la royale); La Chapelle Royale (EL, Regia Capella) (Nègre 1991 et al.).

La Chapelle + nome di un santo (varie);

pochi sono i diminutivi:

Capellette (LG), La Capellette (PdC, TG, Lot, Bouches-du-Rhône), la Chapellette (AL, HL, LI, Somme); La Chapelotte (HS, Cher, oil chapelotte, dim. di chapelle), Chapelon (Loiret, oil chapelle + suff. dim -on) (Nègre 1991: 1506); variante gascona: La Capère (HP) (Nègre 1991 et al.).

Nel territorio germanofono, CAPELLA ha dato:

Kappelen (HR) (Chapellon 1144, Kappel 1303) (Vial 1983: 225 e Nègre 1991), Cappel (Moselle) (Capplen, 1544), fiamm. La Cappelle Grande (Nord), Cappelle Brouck (Nord), 'broek' = marais, Oost Cappel (Nord) (Ostcapellam 1178-1200, fiamm. Oost = de l'est $+\mathrm{kapel}$ ); West Cappel (Nord), nouveau nom d'Arnouds Capple, fiamm. west $=$ ouest.

\section{Cella}

Cella è stata abbastanza produttiva nella toponomastica italiana, con il significato di 'cappella', 'oratorio', 'chiesuola campestre', 'abituro monastico', in senso più esteso 'piccola azienda agraria benedettina'; in più casi, cella può essere intesa come 'grotta, cavità, caverna', 'luogo di conservazione delle derrate'. Cat.: "edifici (religiosi) e annessi” (Cassi e Marcaccini 1998: s.v.). [ < CELLA, origin. 'cella', 'dispensa', cantina', poi 'tempietto'; 'luogo destinato al culto divino']. Ecco una selezione di toponimi italiani:

Cella (CR-FO-RE-VT-CZ), Celle (CN-RV), Cella,-e + det. (vari), Cellasco (Valle Castellana, TE), Cellele (CE), Celletta (PV-CN-FC-FE-PU-BO), Cellina (Leggiuno VA), Cellini (LT-RV-AN-FR), Cellito (Pietracamela - TE), Cellole (FI-SI-CE), Ceola (Malo - VC, San Bonifacio - VR), Ceole (Arco - TN), Ceoli (Vinci e Vodo Cadore BL), Ceule (Lucca), Cévoli (S.Giovanni alla Vena, Vico Pisano), Celiàtica (BS), Cincèlli (Quarata-AR), Cellatica (BS), Cellena (GR) (DT et al.). 
Celle in fr., come in it., designava all'inizio 'una cameretta, una celletta', poi, nel corso dell'alto Medioevo il suo significato si è evoluto in 'cantina' e finirà infine con il designare un romitaggio e talvolta un piccolo monastero, in seguito al raggruppamento degli eremiti nell'epoca carolingia; il termine è molto presente nella toponomastica del territorio francese.

«Parmi les premiers toponymes nés du christianisme, les plus anciens sont formés sur le latin CELLA, mot qui sera évincé par le latin écclesiastique MONASTERIUM aux VI-VIII siècles. Parmi les toponymes dont les attestations sont antérieures à 900, nous trouvons: Celle-sur-Aisne (Aisne, Cellam, VI s.), La Celle-sur-Seine (SM, Cellas 528), Celles (Hérault, Cella en 804; CA, Dor, A), La Celle-saint-Cloud (YV, Cella quae dicitur Villaris 768), Lacelle (CO, Cella 872)» (Gendrom 2003: 146 et al.).

\section{Ecco altri toponimi:}

La Celle $e^{11}$ (Aisne, Celle Gislefridi, vers 840; Cher, Celle, 1150), Cère (Landes), Chelle (HP), Chelles-Debat (HP, debat = au dessus), Chelles-Spou (HP, gasc. espon [< SPONDA, 'versant, talus, limite, talus faisant limite'], Sère (G, HP, transcrit le gasc. cèra, qui vient du latin CELLA, avec le sens d'ermitage, petit monastère) (Fénié e Fénié 2006: 242 et al.).

A partire dal locativo (abl. plurale) CELLIS, abbiamo avuto:

Ceaux, Ceaux + det. (Manche, V, HL) e alcuni Sceaux. Anche Selles ${ }^{12}$ (Eure, Marne, PdC, HS) viene da CELLA, ma altrove (Marne, PdC, HS) viene piuttosto da SAAL (ted., in passato 'grande casa', oggi 'sala') (Vial 1983: 225);

tra le forme con l'articolo agglutinato, incontriamo, oltre al già citato Lacelle:

Lascelle (CA), Lecelles (PdC), Léchelle (PdC), Leschelle (Aisne), Lasseille (HA, SaintJean-Lasseille, catalano Sant Joan la Cella);

con ripetizione dell'articolo, "due à un oubli du sens originel du nom” (Lejeune 2002: 82):

Lalacelle (Orne, Cella, IX sec.);

celle compare anche unita all'aggettivo, come nei seguenti toponimi:

11 In Provenza-Alpi-Costa Azzurra La Celle designava un’abbazia femminile (Fénié e Fénié 2002: 168).

12 I discendenti di CELLA conservano in genere la $\underline{c}$ etimologica, pochi sono i casi in cui compare la forma selle/selles (Gendrom 2003: 47). 
con NOVA in: Naucelle(s) (A, CA, Aveyron), Navacelles (Gard), Neufchelles (Oise), Niozelles (AHP), Novacelles (PdD); con BELLA in: Baucels (Moulès-Her., 820, Bella Cella super fluvium Agotis, 1293, de Baucellis);

il diminutivo CELLULA spiega:

Cellule (PdD, 1401 La Cellule SB), La Ce(l)lette (Cher, 1252 Celleta, Creuse, PdD, CO, Loiret) e Cellettes (Char., L.et C.) (Vial 1983: 227 et al.);

in lingua tedesca, CELLA ha dato zell $^{13}$ :

Rimbachzell (HR, germ. Rindr, 'moglie di Odino' e bach 'ruscello, già Cella Sancti Petri, 817; PdD), Zellwiller (BR, bas latin 'villare', lett. 'frazione sorta inorno a un romitaggio'. In fr. si potrebbe tradurre come Villar-l'Hermitage) (Schweitzer 2001: 43 e Vial 1983: 227); Zell (Alsazia) è stata un'antica designazione (a. 1441) di Labaroche (HR, Festum en 1077, Ecclesia Celle en 1302, Zell en 1441, Payonzelleen 1564, Parochus loci Zell, vulgo La baroche XviIe), parrocchia che si è costituita effettivamente attorno ad un monastero.

\section{Claustrum}

It. Chiostro, s. m. 'convento, monastero' (av. 1294, Guittone), 'portico d'un convento, d'una chiesa' (fine sec. XII, Novellino). (DELI s.v.) Cat.base: "cortile di convento o monastero circondato da porticati” (Cassi 1998: s.v.). [< CLAUSTRUM, orig. 'serratura,' 'cancello', 'luogo chiuso'; mediev. relig. 'clausura'].

In Italia, Chiostro (solo 12 toponimi), è presente più volte solo in Toscana e una sola volta in Umbria:

\section{Chiostro Peccioli, C.Chiostro, Il Chiostro, La Chiostra [pl. Claustra].}

In Francia, v. fr. cloistre $^{14}$ (v. 1100), clostre (v. 1165), [< LT. CLAUSTRUM, soprattutto al pl. ClAUSTRA]: 'chiostro', 'parte di un monastero', poi 'cenobio', monastero', talvolta anche 'recinzione a palizzata d'una fortificazione'. «Le cloître désignait la partie du monastère interdite au profane, plus spécialement la galerie couverte d'une cour intérieure, puis le monastère lui-même» (Gendrom 2003: 147).

Cloitre (V, Claustrae 938), Chiostro (Corse), Clastre, Clastro (= presbytère, Aix-enProvence); clastra équivalent occitan de cloître, est représenté par La Clastre (Gard, DR), Les Clastres (HL), Clost (= Cloître, clôture, Bretagne), Kloastr (= Cloître, Bretagne, pl. en -ou), Encloitre (= monastère, anc. fr.), L'Encloitre à Rouziers-de-Touraine (IL,

13 In Germania ci sono presenze di celle/zell: Celle, città nella Bassa Sassonia, 1292 nome di Neuncelle; Zell nel Baden-W. e nella Renania-Palatinato; anche in Austria incontriamo Zell, centro della Carinzia.

14 L'evoluzione -o- >-oi- è prob.dovuta all'influenza de ${ }^{*}$ cloison, la forma regolare è clostre (DHLF s.v.). 
Claustrum 1272), Lencloitre (V, Claustrum Jarundie 1265, Lencloytre en Jaronde 1303) (Gendrom 2003: 147).

\section{Oratorium}

It. oratorio, s.m. 'Luogo dove si prega', 'edificio o piccolo edificio, spesso annesso a chiese o conventi, per le riunioni religiose' (av. 1348, G. Villani). 'La parola fu adoperata dapprima a indicare un luogo di preghiera e di raccoglimento dove convenivano uomini d'ogni condizione sociale per esercizi di pietà' (Enc. it. XXV: 438). [Lt. tardo ORATORIU(M), derivato di ORARE 'pregare'].

In Italia, sul sito dell'IGMI figurano una trentina di Oratorio, tutti con determinante, prevalentemente localizzati nelle regioni del Nord.

In Francia, dove «désignait sans doute des lieux de culte d'importance secondaire, plus tard érigés en paroisses, puis en commune» (Vial 1983: 230), troviamo numerosi derivati dal lat. ORATORIUM: la forma normale è Oradour (CA, Char, HV), forma primitiva deformata dall'evoluzione fonetica:

Arceau (Anjou), Auradou (LG), Oreor, Oroir (anc. franç.) e ancora Ozoir, Ouzoir, Orrouer, «églises situées sur le passage de voies anciennes. Au nord de la Loire, aboutit à ${ }^{*} \mathrm{Oreu},{ }^{*} \mathrm{Orou},{ }^{*} \mathrm{Ourou}$ avec, dans certains cas, agglutination de l'article (a volte ripetuto): Le Loreur (Manche 1162, de Loratorio)» (Nègre 1991 s.v.); Loreux (LC, Loroir XIII s.), (Le) Louroux (ML, IL, AL, Nièvre, etc.), Oroux (Deux-Sèvres) et bien d'autres. Yrouerre (89), forme moins bourguignonne, prob. attirée par les noms de Tonnerre et Auxerre. Dans le Croissant, Lourdoueix (forma intermedia tra occ.oradour e oil oreoir, con conservazione della dentale -d-) prononcé Lourdoué; Lourdoueix-Saint-Michel (Indre, Oratorium Sancti Michaelis, 1154) et Lourdoueix-Saint-Pierre (Creuse, de Oratorio Sancti Petri, 1154); Othoigü (P. Basq.); Tipedi = chapelle et oratoire (Bretagne) (Nègre 1991 et al.).

Spero di essere riuscito a mostrare, sia pure in modo sommario e semplificativo, come la capillare diffusione della toponomastica religiosa medievale ${ }^{15}$, costituisca ancora oggi una rappresentazione viva della spiritualità cristiana, della romanità e della

15 A titolo di esempio riporto alcuni tipi toponimici dell'ambito religioso spagnolo che confermano il volto sostanzialmente unitario della toponomastica cristiana del nostro continente: "BAsílICA con nombres de pueblo como Baselga o Basella, CAMPANA (Campanario, Campana), CAPIlla (Capellades, Capilla), CRUZ (en especial, los muchos Santa Cruz), ERMita (Fresno y la Ermita, Ermida, Hermida), Iglesia (Iglesia-Rubia, Siete Iglesias, Iglesuela, Elejalde, Elizondo, Grijoa, Grijota, Grijalba, Grisuela), MONASTERIo (Monasteruelo, Mosteiro, Monistrol, Mosteruelos), PARroquia (Parrocha...)" (DGHE:132). Ritengo che la situazione non sia troppo diversa per la toponomastica profana. Ce lo conferma Ottavio Lurati in un suo originale contributo sulla toponomastica laica dell'Europa, in cui parla di "mentalità e identità europea nella lettura del territorio", presentando alcune serie toponimiche tipo: 'Montalbano/ Montauban/ Montalbán/ Montalbä', 'Belmonte/ Belmont/ Beaumont/Schönenberg,' 'Villafranca/Villefranche/ Villa Franca/ Freistadt' e altre ancora, che caratterizzano diverse zone europee (Lurati 2002). 
romanicità, e testimoni che "forse mai il nostro continente è stato così unito e interdipendente come nell'epoca medievale” (Montanelli 2003: 5).

\section{Abbreviazioni}

a. $/$ ant. $=$ antico, agg. $=$ aggettivo, av. $=$ ante, cad. $=$ cadorino, cat. $=$ categoria, dim. $=$ diminutivo, $\mathrm{f} .=$ femminile, $\mathrm{f} . / \mathrm{fr} .=$ francese, fiamm. $=$ fiammingo, friul. $=$ friulano, gen. $=$ genovese, germ . = germanico, gr. $=$ greco, it. $=$ italiano, lad. $=$ ladino, ling. = linguistica, $\mathrm{m} .=$ maschile, med. $/$ mediev. $=$ medievale, n. $_{\text {. }}$ nome, occ. $=$ occitano, $\mathrm{p} . / \mathrm{pp} .=$ pagina, $-\mathrm{e}$, piem. $=$ piemontese, pis . $=$ pisano, pl. $=$ plurale, $\mathrm{s} .=$ sostantivo, s.v. $=$ sotto voce,, $\mathrm{sec} .=$ secolo, sing. $=$ singolare, $\mathrm{sp} .=$ spagnolo, suff. $=$ suffisso, ted.$=$ tedesco, top.$=$ toponimo, vegl. $=$ veglioto

\section{Bibliografia/ Sitografia}

Aebischer, P. 1965. L’Antécédent d'ecclesia sur basilica. In Studi in onore di Alfredo Schiaffini, 6-32. Roma: Edizioni dell'Ateneo.

Battaglia, S. 1961. Grande dizionario della lingua italiana, 1961 e segg. Torino: UTET.

Cassi, L. e P. Marcaccini. 1998. Toponomastica, beni culturali e ambientali. Gli "indicatori geografici” per un loro censimento. Memorie della Società Geografica Italiana, vol. LVI. Roma: Società Geografica Italiana.

Dauzat, A. e Ch. Rostaing. 1978. Dictionnaire étymologique des noms de lieux en France. Paris: Librairie Guénégaud.

De Santis, P. 2009 [2007]. Gli agiotoponimi nella Roma tardoantica. In L'onomastica di Roma. Ventotto secoli di nomi. Atti del Convegno (Roma 19-21 aprile 2007), a cura di Enzo Caffarelli e Paolo Poccetti, 109-122. Quaderni di RIOn 2 (2009). Roma: Società Editrice Romana.

DELI = Cortelazzo, M.e P.Zolli. 1979-88. Dizionario etimologico della lingua italiana. 5 voll. Bologna: Zanichelli.

DGHE = García Borrón, J.-P. 2013. Diccionario geografico de hagiotoponimia espańola. Barcelona: Universitat de Barcelona.

DISC = Sabatini, F.e V.Coletti. 2003. Dizionario della lingua italiana 2004. Milano: Rizzoli-Larousse.

DT = Gasca Queirazza, G., C. Marcato, G.B. Pellegrini, G.P. Sicardi G. e A. Rossebastiano. 1990 (rist. 2010). Dizionario di toponomastica. Storia e significato dei nomi geografici italiani. Torino, UTET.

Fénié B.e J.J. Fénié. 2002. Toponymie provençale. Luçon: Éditions Sud Ouest Université.

Fénié B.e J.J. Fénié. 2003. Toponymie nord-occitane. Périgord, Limousin, Auvergne, Vivarais, Dauphiné. Luçon: Éditions Sud Ouest Université.

Fénié B.e J.J. Fénié. 2006. Toponymie gasconne. Luçon: Éditions Sud Ouest Université.

Fuschi, M. 2008 [2007]. I luoghi della sacralità cristiana nella toponomastica. In Toponomastica italiana: l'eredità storica e le nuove tendenze: atti della Giornata di studio, Pescara 13 dicembre 2007, organizzata da: Università G. d’Annunzio di Chieti-Pescara, a cura di M. Fuschi e G. Massimi, 287-301. Memorie della Società Geografica Italiana Roma: Società Geografica Italiana.

Gendrom, S. 2003. Les noms des lieux en France. Essai de toponymie. Collection des Hesperides. Paris: éd. Errance.

GRADIT = De Mauro, T. 1999-2007. Grande dizionario dell'uso, 8 voll. Torino: UTET. 
Jaccard, H. 1978. Origine des noms de lieux habités et des lieux dits de la Suisse romande. Genève: Éd. Slatkine.

Lejeune, S. 2002. La religion dans la Toponymie. CFC (N.173-174 Sept.-Dec.2002). http:// www.lecfc.fr/ new/articles/173-article-9.pdf (consultato il 25 luglio 2017).

Longnon, A. 1920-29. Les noms de lieu de la France. Paris: Librairie Ancienne Honoré Champion. LUI = Lessico Universale Italiano. 1968-86. Roma: Istituto dell'Enciclopedia Italiana.

Lurati, O. 2002. Identità toponomastica. Tratti incisivi della mentalità europea nella lettura del territorio. L'Erasmo. Bimestrale della civiltà europea. N.10 luglio-agosto 2002: 36-42. Roma: Biblioteca del Senato (Stampa Mondadori).

Montanelli, I. 2003. Storia d'Italia. Vol.I. Milano: RCS Quotidiani SpA.

Moreau, J. 1972. Dictionnaire géographique historique de la Gaule et de la France. Paris: éd. A.et J.Picard.

Nègre, E. 1991. Toponymie générale de la France. (3 vol). Genève: Droz.

Pellegrini, G.B. 1990. Toponomastica italiana: 10.000 nomi di città, paesi, frazioni, regioni, contrade, fumi, monti, spiegati nella loro origine e storia. Milano: Hoepli.

Pop, S. 1966. Recueil posthume de linguistique et dialectologie. Acta Philologica. Tomus IV. Societas Academica Română. Gembloux: Éd. Duculot.

RIOn = Rivista Italiana di Onomastica .

$\mathrm{RLR}=$ Revue de linguistique romane.

Rossebastiano, A. 2007. Gli antroponimi per la caratterizzazione del territorio piemontese. In Atti 11^ Conferenza Nazionale ASITA, Centro Congressi Lingotto, Torino, 6-9 novembre 2007. http:// www.asita.it/Asita2007/Pdf/123pdf (consultato il 15 giugno 2017).

Ruggieri, R.M. 1975. Romanità e cristianesimo nell'Europa medievale. Aspetti e problemi. Roma: Edizioni Studium.

Schweitzer, J. 2001. La toponymie alsacienne. Paris: Éditions Jean-Paul Gisserot.

Tagliavini, C. 1982. Le origini delle lingue neolatine. Bologna: Pàtron.

Taverdet, G. 1994. Noms de lieux de Bourgogne. Paris: Ed. Bonneton.

Vial, É. 1983. Les noms de villes et de villages. Collection «Les français retrouvé». Paris: Belin.

Vincent, A. 1937. Toponimie de la France. Bruxelles: Librairie générale.

treccani online $=h t t p / / w w w . t r e c c a n i . i t /$ vocabolario

http://www.cnrtl.fr/definition/Autel

http://tlio.ovi.cnr.it/TLIO/

http://cnrt.fr./definition/Autel? 